\title{
OFDM Synchronization Improved Algorithm Based on Cyclic Prefix
}

\author{
T.Y. Zhang, G.F. Chen, X.C. Zhang \\ School of Electronic \& Information Engineering \\ Changchun University of Science and Technology \\ Changchun, China
}

\begin{abstract}
The current study synchronization algorithm based on cyclic prefix, requirements of cyclic prefix length cannot be greater than OFDM the 1/4 of the symbol timing synchronization, otherwise, the system timing synchronization error will be significant. Aiming at this problem, this paper again by changing the training sequence structure, puts forward the improved synchronization algorithm, consider the impact of the correlation coefficient on timing synchronous, by an estimate of the correlation coefficient, reducing the factors that affect the performance of the ML timing synchronization algorithm; in the frequency offset estimation, using the set of related ideas to eliminate the drawbacks of ML algorithm performance in multipath channel low, through the simulation verified the effectiveness of the algorithm.
\end{abstract}

Keywords-OFDM; synchronization; cyclic prefix

\section{INTRODUCTION}

By using the method of inserting the training sequence, the system timing synchronization can be achieved very good results. Regardless of the SC algorithm or Minn algorithm and Park algorithm, are increasing at least one data symbol sequence of the same length between the OFDM data symbols and cyclic prefix, broadband and power of the system has a lot of waste, the effectiveness of the system is also affected by a lot [1]. If not through the insertion sequence help system to achieve timing synchronization, then only using the cyclic prefix information of symbols information to achieve.

The earliest use of cyclic prefix to achieve timing synchronization system is Jan-Jaap van de Beek, he proposed maximu m like lihood estimation(ML algorithm) [2] in 1997, ML algorithm is mainly using OFDM symbol cyclic prefix information and maximum likelihood estimate method to realize the combination of system synchronization, so there is no need to add any sequence to help. ML algorithm solve the problem of the validity of the system, it has become a classic algorithm based on cyclic prefix algorithm. However, the current study synchronization algorithm based on cyclic prefix, requirements of cyclic prefix length cannot be greater than OFDM the $1 / 4$ of the symbol timing synchronization, otherwise, the system timing synchronization error will be significant. Aiming at this problem, this paper again by changing the training sequence structure, and puts forward the improved synchronization algorith $\mathrm{m}$ [3]. Solve the problem in improving system performance basis.

\section{IMPROVED TIMING ESTIMATION ALGORITHM AND SIMULATIONANALYSIS}

When we analyze the existing ML algorithm, the correlation $\rho$ coefficient usually set for a fixed amount, but in the realization of the communication system, the correlation coefficient is not only related with the system SNR, but also related with the sample time $k$, so the $\rho$ as a fixed amount inevitably will affect the performance of the system [4].

Improved timing estimation algorithm proposed in this paper is to consider the correlation coefficient $\rho$ as a variable into the determinate likelihood function. Therefore, the algorithm should be estimated for the correlation coefficient $\rho$. The maximu m likelihood function:

$$
\begin{gathered}
\Lambda(d, \Delta f)=\sum_{k=d}^{d+L-1} \frac{2 \rho \operatorname{Re}\left\{e^{j 2 \pi \Delta f} r(k) r^{*}(k+N)\right\}-\rho^{2}\left(|r(k)|^{2}+|r(k+N)|^{2}\right)}{\left(\sigma_{s}^{2}+\sigma_{n}^{2}\right)\left(1-\rho^{2}\right)}-\log \left(1-\rho^{2}\right) \\
=\sum_{k=d}^{d+L-1} \frac{2\left(\rho \lambda(k)-\rho^{2} \phi(k)\right)}{\left(\sigma_{\tilde{x}}^{2}+\sigma_{\omega}^{2}\right)\left(1-\rho^{2}\right)}-\log \left(1-\rho^{2}\right)
\end{gathered}
$$

Among them:

$$
\begin{aligned}
& \lambda(k)=\operatorname{Re}\left\{e^{j 2 \pi \varepsilon} r(k) r^{*}(k+N)\right\} \\
& \phi(k)=|r(k)|^{2}+\left|r(k+N)^{2}\right|
\end{aligned}
$$

The likelihood function is not only a function of $d$ or $\Delta f$, but also the function of $\rho$. But $\rho$ also depends on the sample time $k$. Therefore, the likelihood function is very complex. To the maximum value of the likelihood function, we do not directly make $\Lambda(d, \Delta f)$ biggest, but to make the formula (2) Maximum [5]:

$$
\Lambda_{k}(d, \Delta f)=\frac{2\left(\rho \lambda(k)-\rho^{2} \phi(k)\right)}{\left(\sigma_{\tilde{x}}^{2}+\sigma_{\omega}^{2}\right)\left(1-\rho^{2}\right)}-\log \left(1-\rho^{2}\right)
$$

The formula (2) derivative of $\rho$ :

$\frac{d \Lambda_{k}}{d \rho}=\frac{-2}{\left(\sigma_{s}^{2}+\sigma_{n}^{2}\right)}\left\{\left(\sigma_{s}^{2}+\sigma_{n}^{2}\right) \rho^{3}-\lambda(k) \rho^{2}+\left[2 \phi(k)-\left(\sigma_{s}^{2}+\sigma_{n}^{2}\right)\right] \rho-\lambda(k)\right\}$

For Clearly visible, $(d, \Delta f)$ not to consider, by solving the real roots of following equation, get maximum likelihood estimate $\hat{\rho}$ of $\rho[6]$ :

$$
\left(\sigma_{s}^{2}+\sigma_{n}^{2}\right) \rho^{3}-\lambda(k) \rho^{2}+\left[2 \phi(k)-\left(\sigma_{s}^{2}+\sigma_{n}^{2}\right)\right] \rho-\lambda(k)=0
$$

Among them:

$$
\phi(k) \approx \sigma_{s}^{2}+\sigma_{n}^{2}
$$

Therefore, the equation (4) can be simplified as: 


$$
\phi(k) \rho^{3}-\lambda(k) \rho^{2}+\phi(k) \rho-\lambda(k)=0
$$

After the factorization:

$$
\left(\rho^{2}+1\right)(\phi(k) \rho-\lambda(k))=0
$$

Maximum likelihood estimation $\rho$ of correlation coefficient $\hat{\rho}$

$$
\hat{\rho}=\frac{\lambda(k)}{\phi(k)}
$$
is:

$\hat{\rho}$ into the formula (2), the maximum likelihood function

$$
\Lambda(\theta, \varepsilon)=-\log \left(1-\frac{\lambda^{2}(k)}{\phi^{2}(k)}\right)
$$

Based on the above analysis, for the simulation parameters are set as follows:

TABLE I. SYNCHRONOUS SIMULATION PARAMETERS OF THE IMPROVED ALGORITHM

\begin{tabular}{|l|l|}
\hline Item & Parameters \\
\hline Channel selection & Gaussian channel \\
\hline Carrier length & $\mathrm{N}=1024$ \\
\hline Cyclic prefix length & $\mathrm{L}=120$ \\
\hline Timing deviation sampling number & 100 \\
\hline SNR & $15 \mathrm{~dB}$ \\
\hline CFO & $\mathcal{E}=0.25$ \\
\hline
\end{tabular}

The simulation results are shown in Figure 1. The red curve in the graph is ML algorithm of timing synchronization curve, the blue one is new algorithm of timing synchronization curve. The peak of new algorithm is much larger than the ML algorithm at the position of the timing In this paper, and the curve is more acute.

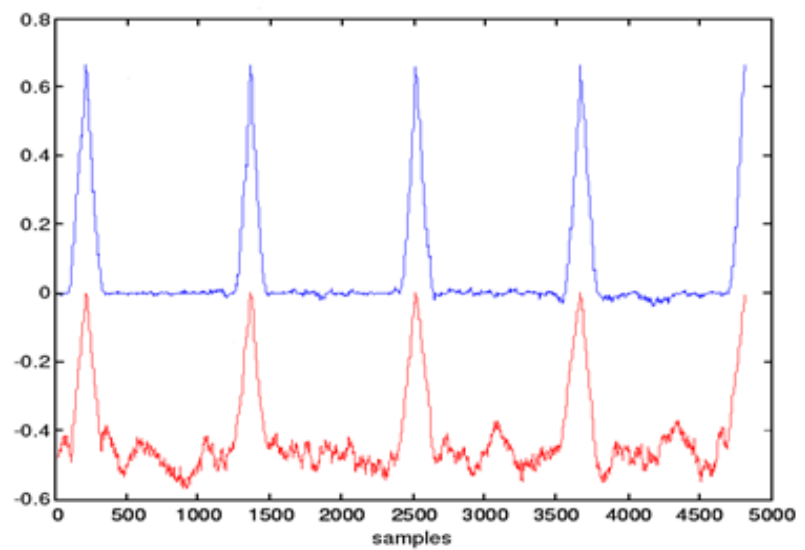

FIGURE I. PERFORMANCE COMPARISON OFTWO ALGORITHMS

From the simulation results, we see that although both algorithms have achieved a peak, but the peak of the new algorithm are bigger. When the new algorith $m$ has greater peak, it did not produce any amplitude peak. For the new algorithm, the synchronization performance of the received signal of the strong interference is better. This also improves the system's immunity to some extent.

\section{IMPROVED FREQUENCY OFFSET ESTIMATION ALGORITHM AND SIMULATION ANALYSIS}

Through the above analysis, the ML algorithm through one by one signal to synchronize, in this way the advantage in the Gauss channel is more outstanding. In multipath channel, cyclic prefix of the OFDM symbol is vulnerable to interference. The synchronization performance of the ML algorithm is significantly decreased [7]. In order to solve this problem, we used carrier frequency offset estimation of set related thoughts. Sets related expand Operating range to $\mathrm{M}$ symbols, it through M symbol estimates obtained after sampling average value in a multipath environment is relatively more accurate.

A receiving end to receive the $M$-th signal is $R, R$ can be expressed as:

$$
r_{m}(n)=e^{j \frac{2 \pi \varepsilon \varepsilon}{N}} x_{m}(n-d)+\omega(n)
$$

Signal away from the $\mathrm{m}$-th signal about $\mathrm{N}$ samples points can be expressed as:

$$
r_{m}(n+N)=x_{m}(n+N-d) e^{j \frac{2 m \varepsilon \varepsilon}{N}} e^{j 2 \pi}+\omega(n+N)
$$

$x_{m}(n)$ is the symbol of the $m$-th of the sender, disregarding the noise [8], then the following holds:

$$
r^{*} \cdot r_{n+N}=e^{j 2 \pi \varepsilon}
$$

So:

$$
\varepsilon=\frac{1}{2 \pi} \arctan \left\{\frac{\operatorname{Im}\left(\sum_{n=0}^{d+L-1} r_{n}^{*} \cdot r_{n+N}\right)}{\operatorname{Re}\left(\sum_{n=0}^{d+L-1} r_{n}^{*} \cdot r_{n+N}\right)}\right\}
$$

Therefore, the function of the carrier Frequency Offset Estimation in this paper can be expressed as:

$$
\varepsilon=\frac{1}{2 \pi} \arctan \left\{\frac{\operatorname{Im}\left(\sum_{M} \sum_{n=0}^{d+L-1} r_{n}^{*} \cdot r_{n+N}\right)}{\operatorname{Re}\left(\sum_{M} \sum_{n=0}^{d+L-1} r_{n}^{*} \cdot r_{n+N}\right)}\right\}
$$

Based on the above analysis, for the simulation parameters are set as follows:

TABLE II. THE SIMULATION PARAMETERS AFTER IMPROVED ALGORIT HM

\begin{tabular}{|l|l|l|}
\hline Item & $\begin{array}{l}\text { Gaussian } \\
\text { channel }\end{array}$ & $\begin{array}{l}\text { Multipath } \\
\text { channel }\end{array}$ \\
\hline Modulation & 64QAM & 64QAM \\
\hline The number of subcarriers & $\mathrm{N}=1024$ & $\mathrm{~N}=1024$ \\
\hline The length of the cyclic prefix & $\mathrm{L}=128$ & $\mathrm{~L}=128$ \\
\hline $\begin{array}{l}\text { The number of symbols about } \\
\text { Extension contains }\end{array}$ & 36 & 36 \\
\hline
\end{tabular}

The graph 2 is the Simulation map of MSE of Frequency Offset in Gaussian channel, the graph 3 is the Simulation map 
of MSE of Frequency Offset in multipath channel. The ML algorithm is the blue curve. The new algorith $m$ is the red curve. From figure 2 we can see that the comparison of two algorithms about Frequency Offset Estimation MSE in Gaussian channel, and improved algorithm is relatively low, synchronized performance was slightly better than ML algorithm, but little difference [9]. In Figure 3, we see a difference of two algorithms on the larger, and the MSE of improved algorithm is less than the MSE of the ML algorithm. This explains the improved algorithm not only has good synchronized performance in Gaussian channel, but also has more obvious advantages of synchronization in the multipath channel.

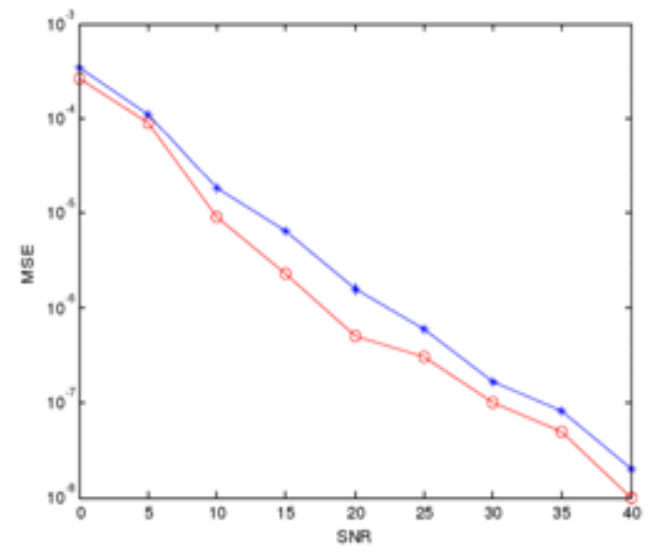

FIGURE II. MSE OF FREQUENCY OFFSET OFTWO ALGORITHMS IN GAUSSIAN CHANNEL

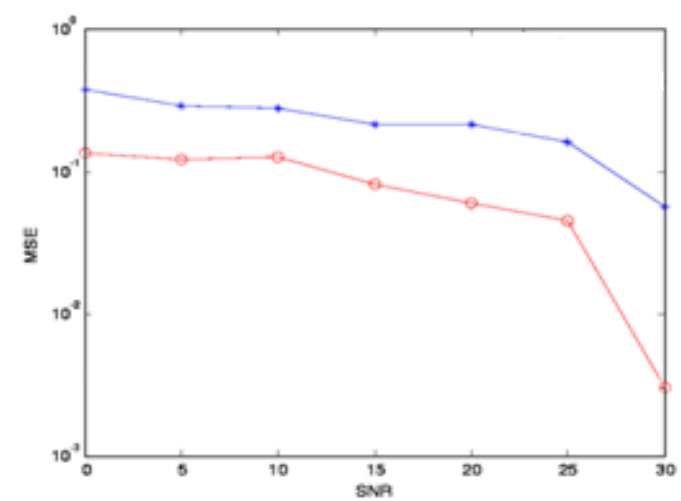

FIGURE III. MSE OF FREQUENCY OFFSET OF TWO ALGORITHMS IN MULTIPAT H CHANNEL

\section{CONCLUSION}

Synchronization algorithm of OFDM is based on cyclic prefix in this paper. By estimating the correlation coeffic ient A, further reduce the factors that affect the performance of timing synchronization of the ML algorithm. In the frequency estimation, use the Sets Related Thought to eliminate Defects of Poor performance of mu ltipath channel in the ML algorith $\mathrm{m}$. Through the simulation comparison, we can see that the improved algorithm not only has good synchronized performance in Gaussian channel, but also has more obvious advantages of synchronization in the multipath channel.

\section{REFERENCES}

[1] Jan-Jaap van de Beek, MagnusSandell. ML estimation of time and frequency offset in OFDM systems[J].IEEE Transactions on signal processing, 1997, 45(7): 1800-1805.

[2] Heung-Gyoon Ryu. Analysis of power efficiencies of polar transmitter and HPA in OFDM communication system[J], International Journal of Electronics, 2014, Vol.101 (2), pp.255-270.

[3] Yongfeng Guan, Tao Xu, Rene Leuken, Manyi Qian. Parallel Channel Estimator and Equalizer for Mobile OFDM Systems[J],Circuits, Systems, and Signal Processing, 2014, Vol.33 (3), pp.839-861.

[4] Yang-Seok Choi, Peter J.Voltz. ML estimation of carrier frequency offset for multicarrier signals in Rayleighfading channels [J].IEEE Transactions on vehicular technology, 2001, 50(2): 644-655.

[5] Hui Wang, Deming Kong, Yan Li. Performance evaluation of (D)APSK modulated coherent optical OFDM system[J], Optical Fiber Technology, 2013, Vol.19 (3), pp.242-249.

[6] Abbas Hasan Kattoush, Waleed Ameen Mahmoud Al-Jawher, Osama Q. Al-Thahab. A Radon-Multiwavelet Based OFDM System Design and Simulation Under Different Channel Conditions[J],Wireless Personal Communications, 2013, Vol.71 (2), pp.857-871.

[7] Ishtiaq Ahmad, Ahmed Iyanda Sulyman, Abdulhameed Alsanie.Spectral broadening effects of high-power amplifiers in MIMO-OFDM relaying channels[J],EURASIP Journal on Wireless Communicat ions and Net working, 2013, Vol.2013 (1), pp.1-14.

[8] Xin-Lin Huang, Gang Wang, Jian Chen.A Novel Haar Wavelet-Based BPSK OFDM System Robust to Spectral Null Channels and with Reduced PAPR[J],W ireless Personal Communications, 2012, Vol.67 (3), pp.599-612.

[9] Zhen hua Yu, Robert J Baxley, Guo Tong Zhou.EVM and achievable data rate analysis of clipped OFDM signals in visible light communication[J],EURASIP Journal on Wireless Communications and Net working, 2012, Vol.2012 (1), pp.1-16. 